\title{
Wheelchair users' anthropometric data: analysis of existent available information
}

\author{
S. Bragança \\ Research and Innovation, Southampton Solent University, Southampton, United Kingdom \\ I. Castellucci \\ Escuela de Kinesiología, Facultad de Medicina, Universidad de Valparaíso, Valparaíso, Chile \\ P. Arezes \\ Centro ALGORITMI, School of Engineering, University of Minho, Guimarães, Portugal
}

\begin{abstract}
Anthropometric data represents the characteristics of a given population and, as such, it varies accordingly. Wheelchair users have, most times, different body characteristics than able-bodied people. These differences can be noticed also in terms of anthropometric data. This paper demonstrates the differences between the results obtained in several studies where anthropometric data of wheelchair users was collected. The results of the comparisons between studies demonstrated that there are some dissimilarities on the data collected. The several studies use different data collection protocols, which compromises the results and the comparability of the results obtained. Hence, it become unclear if the data collected in these studies is accurate and valid enough to be used in other future studies.
\end{abstract}

\section{INTRODUCTION}

Anthropometric data is still limited for the ablebodied population, but it is even scarcer in terms of the structural and functional anthropometric dimensions of wheelchair users (Kozey \& Das 2004).

Research showed that the anthropometric characteristics of wheelchair users are different from the anthropometric characteristics of the able-bodied (Lucero-Duarte et al. 2012).

In fact, anthropometric data varies according to the type of disability - some are characterized by atypical distributions of muscle bulk, bone mass or body stature (Hobson \& Molenbroek 1990). Frequent use of a wheelchair also promotes an overly developed upper body and a more atrophied and weakened lower body (Dingley et al. 2015).

As such, designing workplaces and products for wheelchair users based on the able-bodied population anthropometric is not the most correct procedure to be adopted. According to Das and Kozey (1999), the design guidelines used to develop products for wheelchair users are usually based on timeworn information (Floyd et al. 1966) or extrapolated from the able-bodied population (Pheasant \& Haslegrave 2006). Kozey and Das (2004) and reinforce this by stating that it is not appropriate, nor possible, to properly design a workstation for the wheelchair users' population using information about seated able-bodied workers. Consequently, these authors suggested that there is the need to further investigate the differences between these populations and to also generate more reliable anthropometric data of wheelchair users, which will certainly play an important role in the design of products and spaces. Hobson and Molenbroek (1990) even argue that anthropometric data for the disabled population will need to differentiate between disabilities, and in some cases within disabilities, in order to achieve the desired usefulness. Gonzalez et al. (2012) also referred to the importance of having a European wheelchair users' database to facilitate the development of products, such as clothes.

None of these situations represents the best-case scenario, as this specific population should have its representative anthropometric database. In fact, some previous studies tried to investigate and define the anthropometric characteristics of the disabled population (Urrutia et al. 2015), even trying to compare them with the anthropometric characteristics of the able-bodied population (Goswami et al. 1987). However, it seems to be a difficult task as many of these studies have limited and small sample sizes and do not use the more accurate data collection methods available (such as 3D body scanners).

The main objective of this paper is to compare the data collected in a variety of studies where anthropometric measurements of wheelchair users was gathered. The purpose of this comparison is to understand what are the measurements collected in each study and if there are similarities between the studies, but also to understand if the numerical values obtained are similar and reliable so that they can be used in other future studies. 


\section{METHODOLOGY}

This work is based on the results of a systematic literature review (SLR) that was previously conducted by the authors. This SLR followed a five-step approach and tried to answer the research question: "How can the available literature on anthropometric data collection of wheelchair users be characterized and compared, to allow for a better understanding of the current scenario and to promote the use of data for future studies?"

Three bibliographic databases were selected (ISI Web of Science, Scopus and PubMed) and, using variations of a search string (wheelchair OR "mobility impairment" OR "physical disability" OR "assistive mobility technology" OR "mobility aid") AND ("anthropometr* database" OR anthropometr* OR "body characteristics" OR "physical characteristics" OR "body measurements"), the answer to that research question was found. The SLR resulted in the identification of 40 articles.

For the present study only a selection of these 40 articles has been considered. As the main goal of this study is to compare the data from the different studies, it was extremely important to ensure that the studies were comparable and that there were the largest number of similarities between them as possible. As such, to select the papers to be included, some exclusion criteria were defined:

- papers that did not present the results of the measurements in numbers, but instead in graphs or figures;

- the data collected was regarding children or elderly people;

- the results were clustered in classes or groups, rather than representative of the entire population.

This selection lead to the inclusion of 15 papers in this study.

\section{RESULTS}

The 15 papers selected to this study have several similarities but they are also very different from one another. This difference is especially notorious in the area each paper focused on.

Eight papers were specifically related to the collection of anthropometric data; four focused on sports issues; one on medical issues; and two on product design. As expected this fact impacted not only on the number and type of data collected, but also on the type of statistical analysis performed. Table 1 summarizes this information.

As can be seen, the papers that had the main objective of collecting anthropometric data were the ones where more measurements were gathered and where the percentiles were calculated. A considerable amount of measurements was also collected in the studies that focused on product design. However, and surprisingly, percentiles were not considered.
The other papers, with less focus on ergonomics concerns, collected fewer measurements and analyzed the data only in terms of mean and standard deviation.

Table 1. Characterization of the papers selected for this study.

\begin{tabular}{llll}
\hline T & [ID] Reference & N & Stat.A \\
\hline A & [1] Floyd et al., 1966 & 9 & M; SD; P(5); P(95) \\
& [2] Hosler et al., 1982 & 10 & M; SD; Rg \\
& [3] Goswami et al., 1987 & 12 & M; SD; P(5); P(95) \\
& [4] Jarosz, 1996 & 18 & M; SD; P(5); P(95) \\
& [5] Das and Kozey, 1999 & 16 & M; SD; P(5); P(95); \\
& [6] Paquet and Feathers, & 27 & M; SD; P(5); P(95) \\
& 2004 & & \\
& [7] Barros and Soares, & 15 & M; SD; Rg; Md \\
& 2012 & & \\
& [8] Lucero-Duarte et al., & 14 & M; SD; P(5); P(95) \\
& 2012 & & \\
S & [9] Gass and Camp, 1979 & 7 & M; SD; Rg \\
& [10] Cooper, 1992 & 5 & M; SD \\
& [11] Gil et al., 2015 & 4 & M; SD \\
& [12] Granados et al., & 6 & M; SD \\
& 2015 & & \\
M & [13] Barreto et al., 2009 & 6 & M; SD \\
$D$ & [14] Nitz and Bullock, & 8 & M; SD \\
& 1983 & & \\
& [15] Urrutia et al., 2015 & 10 & I
\end{tabular}

T: Type; [ID]: Study ID number; N Number of Measurements Collected; Stat.A: Statistical Analysis; Anthropometry; S: Sports issues; M: Medical issues; D: Product Design; M: Mean; SD: Standard Deviation; P(5): $5^{\text {th }}$ percentile; P(95): $95^{\text {th }}$ percentile; Rg: Range; Md: Median; I: Individual data.

\subsection{Type of anthropometric measurement}

Regarding the measurements collected across the several papers there are also some discrepancies. The 15 papers collected a total of 80 different anthropometric measurements. Forty-six of these measurements were only collected in a single study, leaving only 34 measurements common to two or more studies.

This indicates that there is an inconsistency in terms of what are the most appropriate measurements to be collected. From this information, it is possible to conclude that there is clearly no specific protocol that studies should use. Instead, the measurements collected are the ones that seem more relevant to the specific application of each study.

Nonetheless, there are a few measurements that seem to be commonly collected by several studies 13 of these measurements were common to at least four studies (Table 2). 
Table 2. Measurements collected in the selected papers

\section{Measurements collected}

1. Abdominal depth, sitting

3. Arm girth*

5. Arm reach forward*

2. Abdominal skinfold

4. Arm girth (contracted)

7. Arm reach overhead from floor, sitting

9. Buttock-popliteal length*

11. Chest girth, sitting

13. Elbow height to chair, sitting

15. Eye height to floor, sitting

17. Hand breadth

19. Hip breadth, sitting*

21. Popliteal height*

23. Shoulder (bideltoid) breadth

25. Shoulder height to chair sitting

27. Shoulder-elbow length

29. Stature*

31. Sum of skinfolds

33. Triceps skinfold lyzed presented large differences according to the study where they were collected, namely: arm reach lateral; stature; knee height; shoulder height to floor, sitting.

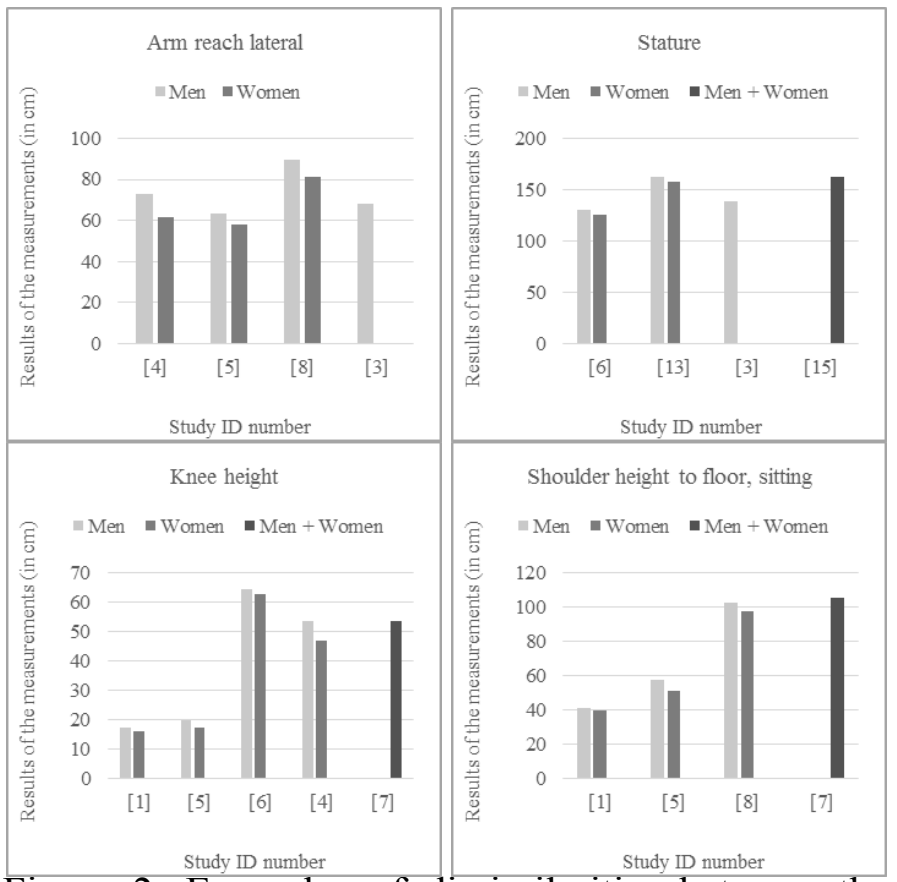

Figure 2. Examples of dissimilarities between the mean values of the measurements collected in different studies.

\section{DISCUSSION}

There are some speculative reasons that can lead to the inconsistencies found in the several studies analyzed. Even though the names given to the measurements collected in each study are more or less similar, it is unclear if they represent the same body parts. Moreover, this is aggravated by the fact that most studies do not give a definition of the measurements to be collected, which makes the process of understanding exactly what is being measured even more difficult. Table 3 demonstrates the differences between the names given to the same measurement and the definitions provided by the studies.

Table 3. Measurements collected in the selected papers.

ID Original name Definition

Arm reach lateral

[3] Arm grasp (Max.) NA

[4] Lateral reach NA

[5] Radial arm reach Sagittal Shoulder marker to the proximal interphalangeal joint

Stature

[8] Lateral arm reach to NA middle finger

[13] Stature NA

[15] Height NA

[6] Overall height Vertical distance from the floor plane to the vertex

[3] Stature NA case in some of the measurements collected As can be seen in Figure 2, four of the measurements ana- 
Table 3. Measurements collected in the selected papers (continued).

ID Original name Definition

Knee height

[7] Height of the knee

Anterior side of the knee floor

[5] Knee height

Sagittal Floor to anterior surface of the thigh (distal end)

[6] Knee height Vertical distance from floor plane to suprapatella landmark

[1] Lower leg length NA

[4] Knee height NA

Shoulder height to floor, sitting

[1] Floor to shoulder NA

[7] Height of the shoul- Acromion - floor der

[8] Shoulder height NA

[5] Shoulder height Sagittal Floor to acromion

ID: Study ID number; NA: Not Available.

As can be noticed, all the studies could be measuring the same body part and giving it different name, but, without a clear definition it is extremely difficult to be certain.

Another possible cause for the discrepancies might be the use of different measuring equipment. Table 4 shows the example of the arm reach lateral measurement, collected by four different studies. As can be observed, not all of the studies used the same data collection technique/equipment and as a result, the measurements obtained are somewhat different.

Table 4. Mean values obtained in different studies for the Arm Reach Lateral measurement.

\begin{tabular}{|c|c|c|c|}
\hline \multirow[t]{2}{*}{ ID } & \multirow[t]{2}{*}{ Measuring devices } & \multicolumn{2}{|c|}{ Mean value in $\mathrm{cm}$} \\
\hline & & Men & Women \\
\hline [3] & $\begin{array}{l}\text { Measuring tape }+ \text { Anthro- } \\
\text { pometer }+ \text { Callipers }\end{array}$ & 67.90 & $\mathrm{~N} / \mathrm{A}$ \\
\hline [4] & $\mathrm{N} / \mathrm{A}$ & 72.75 & 61.72 \\
\hline [5] & Photogrammetry & 63.10 & 58.10 \\
\hline [8] & Measuring tape + Callipers & 89.30 & 81.12 \\
\hline
\end{tabular}

ID: Study ID number.

Furthermore, it was also found that even when the equipment and technology used is the similar, the results vary considerably. Examples of this were found not only for the traditional data collection technique, with measuring tapes and callipers but also for more advanced techniques, as photogrammetry.

In Table 4, it can be noticed that studies [3] and [8] allegedly used the same equipment but the values obtained are very different: a mean of $67.90 \mathrm{~cm}$ in study [3] versus a mean of $89.30 \mathrm{~cm}$ in study [8], which is a difference of $21.40 \mathrm{~cm}$.

In Table 5, three of the studies use photogrammetry but the results are very different: a mean of $19.90 \mathrm{~cm}$ in study [5] versus a mean of $64.50 \mathrm{~cm}$ in study [6], which is a difference of $44.60 \mathrm{~cm}$.
Table 5. Mean values obtained in different studies for the Knee Height measurement.

\begin{tabular}{|c|c|c|c|c|}
\hline \multirow[t]{2}{*}{ ID } & \multirow[t]{2}{*}{ Measuring devices } & \multicolumn{3}{|c|}{ Mean value in $\mathrm{cm}$} \\
\hline & & Men & Women & $\begin{array}{l}\text { Men + } \\
\text { Women }\end{array}$ \\
\hline$[1]$ & Fixed anthropometer & 17.30 & 16.20 & N/A \\
\hline$[4]$ & N/A & 53.65 & 46.83 & N/A \\
\hline$[5]$ & Photogrammetry & 19.90 & 17.20 & N/A \\
\hline [6] & Photogrammetry & 64.50 & 62.80 & N/A \\
\hline [7] & Photogrammetry & $\mathrm{N} / \mathrm{A}$ & $\mathrm{N} / \mathrm{A}$ & 53.45 \\
\hline
\end{tabular}

ID: Study ID number.

Moreover, by comparing all the measurements collected by all the studies it became clear that some of them tended to present measurements with numerical values always smaller than the other studies.

All the measurements presented in study [1] had smaller values that every other study that collected the same measurements. In this particular study, the data was collected with a fixed anthropometer. Any of the other studies used this technique, which might indicate that this is the cause for the discrepancies and that these techniques might not be the most accurate and appropriate one.

On the other hand, study [8] presented, for many of the measurements collected, value higher than the other studies that collected the same measurements. In this case, similar techniques were used by several comparable studies (measuring tapes and callipers) but the results obtained were different.

All of these issues compromise the validity of the results presented in the several studies. The cause of these differences remains unclear. It is recognizable that re-using the data collected in any of these studies might be risky as it is not possible to know which one is the most accurate or representative one.

\section{CONCLUSIONS}

The findings of the present study were very important to clearly understand the current scenario in terms of anthropometric data of wheelchair users.

There are very few measurements that are collected in several studies, which makes it difficult to understand which of the measurements are the most important one to consider.

It can be concluded that the adoption of different measurement protocols result in large differences in the results obtained. Hence, comparing the results obtained in the several studies in extremely difficult as the characteristics are not always the same comparisons would only be reliable if similar protocols were used.

Moreover, this study allowed to understand that using the data collected in previous studies in future studies should be done with caution, if even done at all. It was found that the same measurement collected by different people are sometimes considerably 
different and, as such, it would be complicated to ascertain what would the most reliable data to be used.

Only by comparing the studies side by side is it possible to verify the great dissimilarities between them and the impact that this has on the application of the data.

\section{REFERENCES}

Barreto, F.S. et al. 2009. Nutritional assessment of disabled subjects practitioners of swimming. Revista Brasileira de Medicina do Esporte 15(3): 214-218.

Barros, H.O. \& Soares, M.M. 2012. Using digital photogrammetry to conduct an anthropometric analysis of wheelchair users. Work 41: 4053-4060.

Cooper, R.A. 1992. The contribution of selected anthropometric and physiological variables to $10 \mathrm{~K}$ performance of wheelchair racers: A preliminary study. Journal of Rehabilitation Research and Development 29(3): 29-34.

Das, B. \& Kozey, J.W. 1999. Structural anthropometric measurements for wheelchair mobile adults. Applied Ergonomics 30(5): 385-390.

Dingley, A.A., Pyne, D.B. \& Burkett, B. 2015. Relationships Between Propulsion and Anthropometry in Paralympic Swimmers. International journal of sports physiology and performance 10(8): 978-985.

Floyd, W.F. et al. 1966. A study of the space requirements of wheelchair users. Paraplegia 4(1): 24-37.

Gass, G.C. \& Camp, E.M. 1979. Physiological characteristics of trained australian paraplegic and tetraplegic subjects. Medicine and science in sports and exercise 11(3): 256259.

Gil, S.M. et al. 2015. The Functional Classification and Field Test Performance in Wheelchair Basketball Players. Journal of Human Kinetics 46(1): 219-230.

Gonzalez, J.C. et al. 2012. FASHION-ABLE: Needs and requirements for clothing, footwear and orthotics of consumers groups with highly individualised needs. Proceedings of the 18th International Conference on Engineering, Technology and Innovation: 1-10.

Goswami, A., Ganguli, S. \& Chatterjee, B.B. 1987. Anthropometric characteristics of disabled and normal Indian men. Ergonomics 30(5): 817-823.

Granados, C. et al. 2015. Anthropometry and Performance in Wheelchair Basketball. Journal of Strength and Conditioning Research 29(7): 1812-1820.

Hobson, D.A. \& Molenbroek, J.F.M. 1990. Anthropometry and design for the disabled: Experiences with seating design for the cerebral palsy population. Applied Ergonomics 21(1): 43-54.

Hosler, W.W., Morrow, J.R. \& Boelter, J. 1982. Isokinetic Strength Characteristics Of Men Confined To Wheelchairs. Proceedings of the Human Factors and Ergonomics Society: 181-183.

Jarosz, E. 1996. Determination of the workspace of wheelchair users. International Journal of Industrial Ergonomics 17(2): 123-133.

Kozey, J.W. \& Das, B. 2004. Determination of the normal and maximum reach measures of adult wheelchair users. International Journal of Industrial Ergonomics 33(3), pp.205213.

Lucero-Duarte, K. et al. 2012. Anthropometric data of adult wheelchair users for Mexican population. Work 41: 540810.
Nitz, J.C. \& Bullock, M.I. 1983. Wheelchair design for people with neuromuscular disability. Australian Journal of Physiotherapy 29(2): 43-47.

Paquet, V. \& Feathers, D. 2004. An anthropometric study of manual and powered wheelchair users. International Journal of Industrial Ergonomics 33(3): 191-204.

Pheasant, S. \& Haslegrave, C.M. 2006. Bodyspace: Anthropometry, ergonomics and the design of work, London: CRC Press.

Urrutia, F. et al. 2015. User centered design of a wheelchair based in an anthropometric study. Proceedings of the 2015 IEEE Chilean Conference on Electrical, Electronics Engineering, Information and Communication Technologies: 235-243. 